\title{
Núcleo de Estudos, Pesquisa e Documentação Educação, Sociedade e Cultura (Nedesc) da Faculdade de Educação da Universidade Federal de Goiás: Trajetória e desafios da pesquisa e da formação
}

Center for Studies, Research and Documentation Education, Society and Culture (Nedesc), Faculty of Education, Federal University of Goiás: Research and training trajectories and challenges

\section{João Ferreira de Oliveira' \\ Lúcia Maria de Assis²}

Resumo:

O presente texto analisa a trajetória do Nedesc (Núcleo de Estudos, Pesquisa e Documentação Educação, Sociedade e Cultura), da Faculdade de Educação da Universidade Federal de Goiás, observando as dificuldades metodológicas e organizacionais enfrentadas na formação em Iniciação Científica, Mestrado e Doutorado; e os desafios às tendências de investigação na área da Educação, sobretudo em história da educação, políticas, organização e gestão.

Palavras-chave: Políticas educacionais; Pesquisa; Nedesc/UFG.

\section{Abstract:}

This paper analyzes the trajectory of Nedesc (Center for Studies, Research and Documentation Education, Society and Culture), at Faculty of Education at the Federal University of Goiás, noting the methodological and organizational difficulties faced in education process of students of in Scientific Initiation, Masters and PhD; the challenges and trends of research in education, especially in the history of education, policies, organization and management.

Keywords: Education policies; Research; Nedesc/UFG. 


\section{A criação: os propósitos e a estruturação do Nedesc}

O Núcleo de Estudos, Pesquisa e Documentação Educação, Sociedade e Cultura (Nedesc) foi criado em 1995 a partir da organização da área de estudo e pesquisa, então, denominada Ciências Sociais e Educação da Faculdade de Educação da Universidade Federal de Goiás (FE/UFG)³. Há mais de uma década o Nedesc contribui na qualificação e nos processos de investigação e formação acadêmica com o intuito de vencer os desafios dessa área no campo científico no Brasil. Um espaço estruturado na articulação e na produção do conhecimento em educação, integrando pesquisadores, professores e alunos da graduação e da pós-graduação.

O Nedesc tem por eixo a análise do Estado de Goiás verificando as transformações decorrentes das ações, dos impactos e dos desdobramentos dessas ações na formulação de políticas sociais, particularmente nas políticas educacionais, com especial relevo nos elementos constitutivos e nas intervenções delas decorrentes. Destacam-se como temáticas centrais do Nedesc: o Estado, Educação e Políticas Públicas; a História das Instituições Educacionais, Culturais e Científicas; as Políticas Educacionais, Formação de Professores, Educação Básica, Educação Superior e Gestão Educacional.

Além disso, o Núcleo se estrutura como um centro de documentação em educação. Nessa perspectiva, objetiva o fortalecimento das ações institucionais da FE/UFG e o incremento no intercâmbio interinstitucional por meio do fortalecimento da pesquisa, da graduação e da pósgraduação na região Centro-Oeste. O Nedesc, como espaço de sistematização e ampliação de fontes de documentação, realiza a conexão entre documentação e pesquisa, que, em diálogo entre teoria e método, ressalta a importância histórico-política do conhecimento como uma das formas de compreensão, de aproximação e de explicação da realidade objetiva, que, ao negar os pressupostos da defesa da neutralidade científica, recupera a dupla dimensão da Ciência: a produção histórico-social - fruto de exigências de natureza técnica -, prática ideológica; e a episteme, condicionada às exigências internas de cientificidade. Dessa forma o Núcleo inscrevese como um centro de referência em pesquisa e documentação em educação ao garantir acesso sistemático às fontes primárias e secundárias, subsídio fundamental para o desenvolvimento de pesquisas na área.

O Nedesc abriga vários grupos de pesquisa e de extensão dentro da linha Estado e Políticas Educacionais. Além disso, promove eventos acadêmico-científicos semestralmente, nos quais divulga os resultados das pesquisas, um momento oportuno à realização de intercâmbios com pesquisadores de outras instituições.

Apesar de ter sido concebido como um Núcleo de documentação da memória da educação em Goiás com documentos importantes do patrimônio da Delegacia de Educação do MEC (DEMEC/ GO) e da Faculdade de Educação da UFG, ao longo dos anos 2000, apresentou problemas na organização e no manuseio do acervo existente para pesquisa docente e discente em Iniciação Científica, Mestrado e Doutorado, em virtude, sobretudo das dificuldades em termos de recursos humanos, materiais e financeiros para manutenção e expansão do Núcleo. Além disso, a falta de tratamento adequado dos documentos poderia comprometer a saúde das pessoas que precisavam manuseá-los. Essas dificuldades foram sanadas com a mobilização da coordenação do Núcleo e

3 Para conhecer o Nedesc consultar: http://nedesc.fe.ufg.br/ 
da FE que numa parceria com o Centro de Documentação da UFG, tratou e organizou o acervo. Em decorrência desse processo a digitalização desse material, está sendo efetuada com o intuito de liberar os documentos para o domínio público online, favorecendo o uso e o manuseio virtual aos interessados.

Esta reestruturação, entretanto, não modificou os objetivos do Núcleo em relação ao compromisso inicial com a preservação, utilização e disponibilização de documentos como um contributo à compreensão da história da educação em Goiás, que buscou melhorar as condições de trabalho dos pesquisadores, modernizando o Núcleo com tecnologia.

\section{Reflexões sobre o processo de consolidação do Nedesc}

Ao longo da existência do Nedesc, os professores da área de políticas educacionais e organização escolar da FE/UFG têm vinculado suas pesquisas ao Núcleo, com a finalidade de envolver professores e alunos da graduação, do mestrado e do doutorado em projetos mais abrangentes. Desde o início do Núcleo sentiu-se a necessidade de envolver mais fortemente os professores que atuam na área de história da educação, o que veio ocorrer mais recentemente, embora ainda haja grande dificuldade em integrar os professores e alunos em projetos mais abrangentes. Assim o Núcleo passou a ser um espaço aglutinador de temáticas afins da área de políticas educacionais, organização/gestão escolar e história da educação. Os projetos de pesquisa advindos desses segmentos são apresentados, discutidos e acompanhados, ensejando à participação e o envolvimento do corpo docente e discente e outras pessoas associadas nesses estudos.

Desde o início, o Nedesc apresentou uma forte identificação com a linha de pesquisa Estado e Políticas Educacionais do PPGE/FE/UFG, hoje, denominada: Estado, Políticas e História da Educação. Essa linha foi ampliada para incorporar os estudos em história da educação. A Linha

Analisa o Estado, as transformações decorrentes de suas ações na formulação e implementação de políticas sociais, particularmente das políticas educacionais. Analisa também, no campo da história da educação, a trajetória das instituições escolares, a memória e as representações sociais ligadas a essas instituições. Destacam-se nestas análises, seus elementos constitutivos, desdobramentos em sua formulação e os processos intervencionistas delas decorrentes4.

O Nedesc também tem uma forte vinculação com o Diretório de Pesquisa Estado e Política Educacional, grupo certificado pela UFG e pelo CNPq. Seus líderes, assim como a maior parte dos seus integrantes pertencem ao Nedesc, mas nem todas as pesquisas e pesquisadores do Núcleo estão registradas no diretório, pois a maioria atua na pós-graduação e alguns não veem necessidade de estar em um diretório do CNPq.

\section{As dificuldades metodológicas e organizacionais enfrentadas}

Os projetos de pesquisa desenvolvidos pelo Nedesc associam-se, de modo geral, às temáticas de políticas educacionais, organização, gestão escolar e história da educação. As dificuldades metodológicas são próprias de cada objeto de pesquisa, considerando, sobretudo, os recursos obtidos ou disponíveis; o pessoal envolvido; a abrangência da pesquisa; e a complexidade.

4 Sobre o PPGE e suas linhas de pesquisa consultar: http://ppge.fe.ufg.br/ 
As pesquisas históricas trabalham, normalmente, com objetos advindos da memória educacional: entrevistas, questionários, fotografias, documentos dentre outros. Recentemente esta área tem investido na construção da memória digital, com consequente produção de materiais didáticos importantes para a educação básica, sobretudo na área das ciências sociais (história/geografia).

Já as pesquisas na área de políticas educacionais e organização escolar enfocam, em geral, os estudos nas políticas estaduais, municipais, contemplando também o âmbito das políticas federais. As temáticas, nesse segmento, são abrangentes e estão associadas aos projetos de pesquisa desenvolvidos pelos professores que constituem o Núcleo. Desse modo, não há uma temática única que agregue a todos.

As pesquisas na área de políticas e organização escolar têm uma agenda de objetos de investigação acoplada às políticas governamentais, o que acaba provocando certo presentismo nas investigações e, dessa forma, corre-se o risco de deixar que a agenda governamental de políticas determine os temas a serem pesquisados, ou seja, o Estado acaba por determinar os objetos de estudo mais importantes. Isso também ocorre porque muitos professores e pesquisadores, que estão envolvidos em projetos de extensão, de formação de professores ou consultorias educacionais, passam a utilizar essas experiências na realização de suas pesquisas. Isso provoca maior apropriação e domínio do objeto e, ao mesmo tempo, a ausência do necessário distanciamento para uma boa revisão da literatura e da crítica.

No âmbito no Nedesc, observa-se uma dificuldade, em articular, nas pesquisas históricas e nas pesquisas focadas nas políticas educacionais e organização escolar, o que pode ser atribuído às dificuldades históricas no campo das investigações acadêmicas, no que tange à transposição das barreiras entre as disciplinas. Fala-se muito nas abordagens interdisciplinares, mas, na prática, esse diálogo entre as diferentes áreas ocorre pouco, inclusive nas áreas consideradas afins, como é o caso da política e da história, um desafio no qual vale a pena investir nos próximos projetos do Núcleo.

\section{Os desafios e as tendências para a investigação no campo de atuação do Nedesc}

O Nedesc tem muitos desafios e tendências a pesquisar em educação, principalmente na área de políticas educacionais e organização escolar. Atualmente há distintos agentes que realizam pesquisas na área, dentre os quais se destacam: universidades, órgãos de governos, organismos multilaterais, entidades científicas, sindicatos, organizações não governamentais (ONGs), redes de pesquisa, núcleos, programas de pós-graduação, além dos pesquisadores individuais. Os propósitos dessas pesquisas são diversos: diagnósticos, confirmação de políticas, formação de pesquisadores, divulgação, acompanhamento de políticas, lucro etc. Portanto, estamos num campo de interesses, de conflitos e de múltiplos olhares, perspectivas teóricas, ideologias. Isso implica em fazer uma boa revisão da literatura lendo as entrelinhas do texto, em prestar atenção em afirmações que não apresentam sustentação empírica ou manipulam dados em razão de interesses diversos.

Outro tema importante é o financiamento das pesquisas. A área de Políticas Educacionais conta hoje com muitas fontes, embora boa parte dos estudos e dados a serem utilizados seja definida pelo próprio órgão, o que diminui a autonomia do pesquisador que, mesmo contando com o fomento, não 
consegue aprovação em determinada área de pesquisa como a Coordenação de Aperfeiçoamento de Pessoal de Nível Superior (Capes) e o Conselho Nacional de Desenvolvimento Científico e Tecnológico (CNPq), por exemplo.

Há também uma tendência de se realizar análises de políticas muito recentes que sequer apresentam impactos significativos. Entendemos que as políticas educacionais têm, no mínimo, três momentos: a concepção ou origem, a implantação e os resultados ou impactos. Quase sempre os pesquisadores querem apreender esses três momentos e não conseguem fazer com a devida pertinência, sobretudo porque, para fazer isso, seriam necessários grupos maiores de pesquisa e com mais recursos.

Outro desafio é o de conseguir elevar o grau de comunicação entre o que se pesquisa o que se produz em termos de programas, projetos e ações no campo da educação. Há pouco diálogo entre o mundo acadêmico e o mundo político-educacional que envolve a gestão do ministério da educação, dos órgãos de pesquisa, como Instituto Nacional de Estudos e Pesquisas Educacionais Anísio Teixeira (Inep), Instituto de Pesquisa Econômica Aplicada (IPEA), Instituto Brasileiro de Geografia e Estatística (IBGE), Capes, CNPq, das secretarias de educação e outras. A lógica parece ser distinta e o mesmo ocorrer no insuficiente diálogo entre as instituições que formam professores e as que contratam professores e são responsáveis pela formação continuada.

A abrangência da pesquisa é outro problema a ser resolvido. O que predomina na área são pesquisas pequenas, com um caráter formativo para o pesquisador, mas com resultados que acrescentam pouco na promoção de mudanças mais gerais em relação à problemática específica. Muitas pesquisas são feitas com o único objetivo de formar mestrandos e doutorandos, apresentando impacto relativo em termos de produção de conhecimento novo. A redução do tempo para realização do mestrado e do doutorado contribuiu para intensificar esta tendência.

Há um forte crescimento da pesquisa e na pós-graduação em educação. Somos uma das maiores áreas com mais de 120 programas 5 na Capes. Existe, atualmente, uma forte pressão, com a expansão dos mestrados profissionais, para tornar a produção mais prática e mais aplicada, pois se espera mudar a escola e a prática dos professores por meio desse tipo de intervenção. A área de gestão sofre grande influência dessa lógica, pois o diretor é visto cada vez mais como um gerente, um líder, que deve implementar uma gestão eficaz, eficiente, focada em metas e resultados.

Tais desafios nos movimentam a uma busca permanente de novas possibilidades, novas experiências que ousem desafiar os paradigmas e as tendências dominantes, sobretudo no que diz respeito ao aprofundamento dos temas trabalhados de modo que pesquisas de maior impacto no campo das políticas públicas educacionais venham a ser produzidas e divulgadas pelo Nedesc.

Refletir sobre a trajetória do Nedesc - as dificuldades metodológicas e organizacionais enfrentadas e os desafios e tendências para a investigação na área de educação -, certamente, nos ajuda a compreender os processos de elaboração da pesquisa e os possíveis impactos na área, assim como possibilita refletir sobre processos de formação de estudantes de iniciação científica, de mestrado e de doutorado. Além disso, o campo de pós-graduação e de pesquisa na área de educação é

5 Em 11 de setembro de 2012 a Capes registrava 122 cursos/programas na área de educação. Disponível no site: http://www.capes.gov.br/. Acesso em 11/09/2012. 
composto por múltiplos agentes institucionais e individuais, o que requer um conhecimento mais amplo e totalizar dos desafios dessa área como campo científico no Brasil.

\section{Referências Bibliográficas}

DOURADO, L. F. et al. Gestão escolar democrática: a perspectiva dos dirigentes escolares da rede municipal de ensino de Goiânia-GO. Goiânia: Alternativa, 2003.

DOURADO, L. F.; CATANI, A. M.; OLIVEIRA, J. F. Políticas e gestão da educação superior: transformações recentes e debates atuais. 1. ed. São Paulo / Goiânia: Xama / Alternativa, 2003.

LIBÂNEO, J. C.; OLIVEIRA, J. F.; TOSCHI, M. S. Educação escolar: políticas, estrutura e organização - $4^{a}$ edição. 10ª . ed. São Paulo-SP: Cortez, 2011.

LOUREIRO, W. N. et al. Formação e profissionalização docente. 1. ed. Goiânia-GO: CEGRAF - UFG, 1999. OLIVEIRA, J. F.; BITTAR, M. (Org.). Gestão e políticas da educação. 1ª. ed. Rio de Janeiro-RJ: DP\&A, 2004.

UNIVERSIDADE FEDERAL DE GOIAS. FACULDADE DE EDUCAÇÃO. Núcleo de Estudos, Pesquisa e Documentação Educação, Sociedade e Cultura (Nedesc). Relatório de Atividades 2007-2010. Goiânia, NEDESC/FE/UFG, 2010.

Recebido em Setembro de 2012 Aprovado em Outubro de 2012 